\title{
On $n$-Perfect Rings and Cotorsion Dimension
}

\author{
Driss Bennis and Najib Mahdou \\ Department of Mathematics, Faculty of Science and Technology of Fez, \\ Box 2202, University S. M. Ben Abdellah Fez, Morocco \\ driss_bennis@hotmail.com \\ mahdou@hotmail.com
}

\begin{abstract}
A ring is called $n$-perfect $(n \geq 0)$, if every flat module has projective dimension less or equal than $n$.

In this paper, we show that the $n$-perfectness relates, via homological approach, some homological dimensions of rings. We study $n$-perfectness in some known ring's construction. Finally, several examples of $n$-perfect rings satisfying special conditions are given.
\end{abstract}

Keywords: Cotorsion dimension of modules and rings; $n$-perfect rings; (finitistic) homological dimensions.

2000 Mathematics Subject Classification. 13D02, 13D05, 13D07

\section{Introduction}

Throughout this paper all rings are commutative with identity element and all modules are unitary. For a ring $R$ and an $R$-module $M$, we use $\operatorname{pd}_{R}(M), \operatorname{id}_{R}(M)$, and $\mathrm{fd}_{R}(M)$ to denote, respectively, the classical projective, injective, and flat dimension of $M$. We use $\operatorname{gldim}(R)$ and $\operatorname{wdim}(R)$ to denote, respectively, the classical global and weak dimension of $R$. If $R$ is integral, we denote its quotient field by qf $(R)$.

In [1], Bass proved that the perfect rings are those rings such that every flat module is projective. He links these rings with the finitistic projective dimension of rings. Recall that the finitistic projective dimension of a ring $R$, denoted by $\operatorname{FPD}(R)$, is defined by: $\operatorname{FPD}(R)=\sup \left\{\operatorname{pd}_{R}(M) \mid M R\right.$-module with $\left.\operatorname{pd}_{R}(M)<\infty\right\}$.

From [1, Kaplansky's Theorem, page 466] and [1, Part I, 3. Example (6)], the following are equivalent, for a commutative ring $R$ : 
1. $R$ is perfect;

2. $R$ is a finite direct product of local (not necessarily Noetherian) rings, each with $T$-nilpotent maximal ideal (i.e., if we pick a sequence $a_{1}, a_{2}, \ldots$ of elements in the maximal ideal, then for some index $\left.m, a_{1} a_{2} \ldots a_{m}=0\right)$;

3. $\operatorname{FPD}(R)=0$.

Later, in [10, Proposition 6], Jensen proved that, for a ring $R$, if $\operatorname{FPD}(R)=n$ $(n \geq 0)$, then every flat $R$-module has projective dimension at most $n$. In several situations the Jensen's result implies the desired properties. But, unfortunately, the finitistic projective dimension is not known to be finite only in few cases (for example for Noetherian rings with finite Krull dimension [9, Theorem 3.2.6]), and its finiteness remains an open problem. Then, it seems appropriate to investigate the rings over which every flat module has projective dimension at most $n$, where $n$ is a fixed positive integer. In [7, Definition 1.1], Enochs, Jenda, and López-Ramos called these rings $n$-perfect. These rings were homologically characterized by the cotorsion dimension introduced by Ding and Mao as follows:

Definition 1.1 ([5]) Let $R$ be a ring.

The cotorsion dimension of an $R$-module $M$, denoted by $\operatorname{cd}_{R}(M)$, is the least positive integer $n$ for which $\operatorname{Ext}_{R}^{n+1}(F, C)=0$ for all flat $R$-modules $F$.

The global cotorsion dimension of $R$, denoted by $\mathrm{C}-\operatorname{gldim}(R)$, is the quantity: $\mathrm{C}-\operatorname{gldim}(R)=\sup \left\{\operatorname{cd}_{R}(M) \mid M R-\right.$ module $\}$.

Namely, the modules of cotorsion dimension 0 are the known cotorsion modules (see [14, Definition 3.1.1]). We have:

Proposition $1.2([5]$, Theorem 19.2.5(1)) For a positive integer $n, R$ is $n$-perfect if and only if $\mathrm{C}-\operatorname{gldim}(R) \leq n$.

So, Jensen's result above can be written again as follows:

Proposition 1.3 ([10], Proposition 6) For any $\operatorname{ring} R, \mathrm{C}-\operatorname{gldim}(R) \leq \operatorname{FPD}(R)$.

Early, in [9, page 87], Gruson and Raynaud defined $d(R)$ as the supremum of the projective dimensions of all flat $R$-modules. Then, $d(R)$ coincides with the global cotorsion dimension of $R$. They shortly studied this invariant of rings and they mentioned that Jensen had an example of a ring $R$ that satisfies the strict inequality $\mathrm{C}-\operatorname{gldim}(R)<\operatorname{FPD}(R)$. One of our aims in this paper is to give further concrete examples of rings with the strict inequality, which means that the converse of Jensen's result is not true in general (see Section 4). 
In Section 2, we give some general results on the global cotorsion dimension of rings. Mainly, we extent the inequality $\operatorname{gldim}(R) \leq \mathrm{C}-\operatorname{gldim}(R)+\operatorname{wdim}(R)$ established by Ding and Mao in [5, Theorem 19.2.14] to the finitistic dimensions (see Theorems 2.2 and 2.4).

In Section 3, we investigate $n$-perfectness in some known ring's constructions, such that we compute the global cotorsion dimension of polynomial rings, finite direct products of rings, and $D+M$ rings. This study allows us to give various examples of $n$-perfect rings satisfying special conditions. This is given in Section 4 .

\section{General results}

In [5, Theorem 19.2.14], the global cotorsion dimension of rings is used to relate the weak and the global dimensions as follows:

Theorem 2.1 ([5], Theorem 19.2.14) For any ring $R$, the following inequality $\operatorname{gldim}(R) \leq \mathrm{C}-\operatorname{gldim}(R)+\mathrm{wdim}(R)$ holds true.

In particular:

- If $\mathrm{C}-\operatorname{gldim}(R)=0$ (i.e., $R$ is perfect), then $\operatorname{wdim}(R)=\operatorname{gldim}(R)$.

- If $\operatorname{wdim}(R)=0$ (i.e., $R$ is von Neumann regular), then $\mathrm{C}-\operatorname{gldim}(R)=$ $\operatorname{gldim}(R)$.

The next theorem generalizes this result to the finitistic projective and flat dimensions. Recall that the finitistic flat dimension of $R, \operatorname{FFD}(R)$, is defined as follows: $\operatorname{FFD}(R)=\sup \left\{\operatorname{fd}_{R}(M) \mid M R\right.$-module with $\left.\operatorname{fd}_{R}(M)<\infty\right\}$.

Theorem 2.2 For any ring $R$, the following inequalities $\operatorname{FFD}(R) \leq \operatorname{FPD}(R) \leq$ $\mathrm{C}-\operatorname{gldim}(R)+\mathrm{FFD}(R)$ hold true.

Proof. To prove the inequality $\operatorname{FFD}(R) \leq \operatorname{FPD}(R)$ we can assume that $\operatorname{FPD}(R)=$ $n$ is finite. Consider an $R$-module $M$ with finite flat dimension. From Jensen's Proposition 1.3, $M$ has also finite projective dimension, which is at most $n$. Then, we have $\operatorname{fd}_{R}(M) \leq \operatorname{pd}_{R}(M) \leq n$. This means that $\operatorname{FFD}(R) \leq \operatorname{FPD}(R)$, as desired. Now we prove the second inequality. For that we can assume that $\mathrm{C}-\operatorname{gldim}(R)=$ $n$ and $\operatorname{FFD}(R)=m$ are finite. Consider an $R$-module $M$ with finite projective dimension, then it has finite flat dimension, which is at most $m$. Then, there exists an exact sequence of $R$-modules

$$
0 \rightarrow F \rightarrow P_{m-1} \rightarrow \cdots \rightarrow P_{0} \rightarrow N \rightarrow 0
$$


where $P_{i}$ are projective and $F$ is flat. From Proposition $1.2, \operatorname{pd}_{R}(F) \leq n$. Finally, using the above sequence, we get $\operatorname{pd}_{R}(M) \leq n+m$. This completes the proof.

In Section 4, Example 4.5 shows that the upper bound on the finitistic projective dimension in Theorem 2.2 above is the best possible upper bound.

As mentioned in the introduction, in [9, page 87], $d(R)$ denotes, for a ring $R$, the supremum of the projective dimensions of all flat $R$-modules, then it coincides with the global cotorsion dimension of $R$. The authors mentioned that Jensen constructed an example showing that the inequality $\mathrm{C}-\operatorname{gldim}(R) \leq \mathrm{FPD}(R)$ can be strict. In Section 4 , we give several examples showing that each of the inequalities of both Theorems 2.1 and 2.2 can be strict.

The following gives a situation in which the upper bound on the finitistic projective dimension (and then of the global dimension) in Theorem 2.2 (and Theorem 2.1) is reached. Later, Example 4.5 (and Example 4.2) provides a ring satisfying this situation.

Proposition 2.3 If a ring $R$ satisfies $\operatorname{FFD}(R)=1$ and $\mathrm{C}-\operatorname{gldim}(R)<\operatorname{FPD}(R)<$ $\infty$, then: $\operatorname{FPD}(R)=\mathrm{C}-\operatorname{gldim}(R)+1$.

Proof. Let $\operatorname{FPD}(R)=n<\infty$ for an integer $n \geq 1$. Then, there is an $R$-module $M$ that satisfies $\operatorname{pd}_{R}(M)=n$. Hence, for a short exact sequence of $R$-modules $0 \rightarrow F \rightarrow$ $P \rightarrow M \rightarrow 0$, where $P$ is projective and then $F$ is flat ( $\operatorname{since} \operatorname{FFD}(R)=1$ ), we have $\operatorname{pd}_{R}(F)=n-1$. Then, the desired equality holds since $\mathrm{C}-\operatorname{gldim}(R)<\operatorname{FPD}(R)$.

Recall that the finitistic injective dimension of a ring $R$, denoted by $\operatorname{FID}(R)$, is defined by: $\operatorname{FID}(R)=\sup \left\{\operatorname{id}_{R}(M) \mid M R\right.$-module with $\left.\operatorname{id}_{R}(M)<\infty\right\}$. Similarly, we can define the finitistic cotorsion dimension of a ring $R$, denoted by $\operatorname{FCD}(R)$, as follows: $\operatorname{FCD}(R)=\sup \left\{\operatorname{cd}_{R}(M) \mid M R\right.$-module with $\left.\operatorname{cd}_{R}(M)<\infty\right\}$.

The following result is another extension of Theorem 2.1.

Theorem 2.4 For any ring $R$ with finite weak dimension, the following inequalities $\mathrm{FCD}(R) \leq \mathrm{FID}(R) \leq \mathrm{FCD}(R)+\mathrm{wdim}(R)$ hold true.

The proof involves the following lemma which relates the cotorsion dimension and the injective dimension of modules.

Lemma 2.5 Let $R$ be a ring. For any $R$-module $M$, the following inequalities $\operatorname{cd}(M) \leq \operatorname{id}(M) \leq \operatorname{cd}(M)+\operatorname{wdim}(R)$ hold true. 
Proof. The proof of the first inequality is easy.

To prove the second inequality, we can assume that $\operatorname{cd}(M)=m$ and $\operatorname{wdim}(R)=n$ are finite. Let $N$ be any $R$-module, and consider an exact sequence $0 \rightarrow F \rightarrow$ $P_{n-1} \rightarrow \cdots \rightarrow P_{0} \rightarrow N \rightarrow 0$, where $P_{i}$ are projective modules and then $F$ is a flat module ( since $w \operatorname{dim}(R)=n)$. We have $\operatorname{Ext}^{m+n+1}(N, M) \cong \operatorname{Ext}^{m+1}(F, M)=0$ (since $\operatorname{cd}(M)=m)$. Therefore, $\operatorname{id}(M) \leq m+n$, as desired.

Proof of Theorem 2.4. We prove the inequality $\operatorname{FCD}(R) \leq \operatorname{FID}(R)$. We can suppose that $\operatorname{FID}(R)=n$ is finite. Let $M$ be an $R$-module with finite cotorsion dimension. From Lemma 2.5, $\operatorname{id}(M) \leq \operatorname{cd}(M)+\operatorname{wdim}(R)$ which is finite. Then, $\operatorname{id}(M)$ is finite and so $\operatorname{cd}(M) \leq \operatorname{id}(M) \leq n$, as desired.

We prove the inequality $\operatorname{FID}(R) \leq \operatorname{FCD}(R)+$ wdim $(R)$. We can suppose that $\operatorname{wdim}(R)=n$ and $\operatorname{FCD}(R)=m$ are finite. Let $M$ be an $R$-module with finite injective dimension. Then, from Lemma 2.5, $\operatorname{id}(M) \leq \operatorname{cd}(M)+\mathrm{wdim}(R) \leq m+n$. This implies the desired inequality.

In [9, Remark 3.3.3(1), page 87], the authors ask whether, for an integral Noetherian domain $R$ with quotient field $Q, \operatorname{pd}_{R}(Q)=\mathrm{C}-\operatorname{gldim}(R)$. Next, we analyze some situations in which we have an affirmative answer. But, for non-Noetherian domains, Example 4.3 shows that we have a negative answer (see Remark 4.4).

Proposition 2.6 Let $R$ be a domain which is not a field, and let $Q$ be the quotient field of $R$. Then, the following assertions hold true:

1. If $\mathrm{C}-\operatorname{gldim}(R) \leq 1$, then $\mathrm{C}-\operatorname{gldim}(R)=\operatorname{pd}_{R}(Q)$.

2. If $\mathrm{C}-\operatorname{gldim}(R)=\operatorname{FPD}(R)<\infty$, then $\mathrm{C}-\operatorname{gldim}(R)=\operatorname{pd}_{R}(Q)$.

3. If $\operatorname{gldim}(R)=\operatorname{pd}_{R}(Q)+1$, then $\mathrm{C}-\operatorname{gldim}(R)=\operatorname{pd}_{R}(Q)$.

Proof. 1. Since the quotient field of a domain which is not a field is flat but not projective, we deduce that:

- If $\mathrm{C}-\operatorname{gldim}(R)=0$ (i.e., $R$ is perfect), then $R$ is a field. So, $R=Q$ and $\mathrm{C}-\operatorname{gldim}(R)=\operatorname{pd}_{R}(Q)=0$.

- If $\mathrm{C}-\operatorname{gldim}(R)=1$, then $R \subsetneq Q$ and then $\operatorname{pd}_{R}(Q)=1$, as desired.

2. From (1), we can assume that $0<\mathrm{C}-\operatorname{gldim}(R)=\operatorname{FPD}(R)=n<\infty(n \geq 1)$. Let $F$ be a flat $R$-module such that $\operatorname{pd}_{R}(F)=n$. From [13, Lemma 4.31 and the Remark bellow Theorem 4.33], there exist an index set $I$ and an $R$-module $M$ such 
that the sequence $0 \rightarrow F \rightarrow Q^{(I)} \rightarrow M \rightarrow 0$ is exact. Then, if we suppose that $\operatorname{pd}_{R}(Q)<n$, we obtain that $\operatorname{pd}_{R}(M)=n+1$, which gives a contradiction.

3. We can assume that $\operatorname{pd}_{R}(Q)=n$ for a positive integer $n$. As above, for every flat module, there are an index set $I$ and an $R$-module $M$ such that the sequence $0 \rightarrow F \rightarrow Q^{(I)} \rightarrow M \rightarrow 0$ is exact. From [3, Corollary 2(c) and Corollary 1, page 135], we have $\operatorname{pd}(F) \leq \sup \{\operatorname{pd}(Q), \operatorname{pd}(M)-1\} \leq n\left(\operatorname{since} \operatorname{gldim}(R)=\operatorname{pd}_{R}(Q)+1=\right.$ $n+1)$. This completes the proof.

In Section 4, we construct a ring satisfying (1) and (2) of Proposition 2.6 (see Example 4.2).

\section{$3 n$-Perfectness in some known ring's construc- tions}

In this section, we establish some results concerning the transfer of the $n$-perfectness in some known ring's constructions. These results will be used to construct some desired examples.

We begin by computing the global cotorsion dimension of polynomial rings.

Theorem 3.1 Let $R\left[X_{1}, X_{2}, \ldots, X_{n}\right]$ be the polynomial ring in $n$ indeterminates over a ring $R$. Then, for a positive integer $m, R$ is $m$-perfect if and only if $R\left[X_{1}, X_{2}, \ldots, X_{n}\right]$ is $m+n$-perfect.

In other words, $\mathrm{C}-\operatorname{gldim}\left(R\left[X_{1}, X_{2}, \ldots, X_{n}\right]\right)=\mathrm{C}-\operatorname{gldim}(R)+n$.

Proof. By induction, it suffices to prove the case $n=1$. We write $R\left[X_{1}\right]=R[X]$. Through this proof, we use $M[X]$ to denote the $R[X]$-module $M \otimes_{R} R[X]$.

We prove that $\mathrm{C}-\operatorname{gldim}(R[X])=\mathrm{C}-\operatorname{gldim}(R)+1$.

The inequality $\mathrm{C}-\operatorname{gldim}(R[X]) \leq \mathrm{C}-\operatorname{gldim}(R)+1$ is the same as [7, Example 2.5]. Conversely, assume that $\mathrm{C}-\operatorname{gldim}(R[X])=n+1<\infty$. Let $F$ be a flat $R$-module, then $F[X]$ is a flat $R[X]$-module. Then, $\operatorname{pd}_{R}(F)=\operatorname{pd}_{R}(F[X]) \leq \operatorname{pd}_{R[X]}(F[X]) \leq$ $n+1$. This means that $\mathrm{C}-\operatorname{gldim}(R) \leq n+1$. Assume that $\mathrm{C}-\operatorname{gldim}(R)=n+$ 1. Then, there exists, from [5, Theorem 19.2.5(1)], a flat $R$-module $F$ such that $\operatorname{cd}_{R}(F)=n+1$. Thus, there exists a flat $R$-module $E$ such that $\operatorname{Ext}_{R}^{n+1}(E, F) \neq 0$. From [3, Example 7, page 9], the endomorphism $\mu: F \rightarrow F$, defined by $\mu(f)=X f$, is injective. Then, Rees's Theorem [13, Theorem 9.37] gives:

$$
\operatorname{Ext}_{R[X]}^{n+2}(E, F[X]) \cong \operatorname{Ext}_{R}^{n+1}(E, F) \neq 0 .
$$


From [13, Exercise 9.20, page 258],

$\operatorname{Ext}_{R[X]}^{n+2}(E[X], F[X]) \cong \operatorname{Hom}_{R}\left(R[X], \operatorname{Ext}_{R[X]}^{n+2}(E, F[X])\right) \cong\left(\operatorname{Ext}_{R[X]}^{n+2}(E, F[X])\right)^{\mathbb{N}} \neq 0$.

Then, $\operatorname{cd}_{R[X]}(F[X]) \geq n+2$, which contradicts $\mathrm{C}-\operatorname{gldim}(R[X])=n+1$.

Now, we compute the global cotorsion dimension of a finite direct product of rings. Namely, we extent the obvious fact that a finite direct product of rings is perfect if and only if each of these rings is perfect.

Theorem 3.2 Let $\left\{R_{i}\right\}_{i=1, \ldots, m}$ be a family of rings. Then, for a positive integer $n$, $\prod_{i=1}^{m} R_{i}$ is $n$-perfect if and only if each of $R_{i}$ is n-perfect.

In other words, $\mathrm{C}-\operatorname{gldim}\left(\prod_{i=1}^{m} R_{i}\right)=\sup \left\{\mathrm{C}-\operatorname{gldim}\left(R_{i}\right), 1 \leq i \leq m\right\}$.

Proof. It follows by induction on $m$ and using [12, Lemma 2.5 (2)] and [2, Lemma 3.7].

We end this section with a study of the $n$-perfectness in a particular case of the $D+M$-constructions. These constructions have been proven to be useful in solving many open problems and conjectures in various contexts in ring theory (please see [8. Section 1, Chapter 5]; see also [6] and [12]).

Theorem 3.3 Let $T$ be a domain of the form $K+M$, where $K$ is a field and $M(\neq 0)$ is a maximal ideal of $T$. Let $D$ be a proper subring of $K$ such that qf $(D)=K$. Then, for the subring $R=D+M$ of $T$, we have $\mathrm{C}-\operatorname{gldim}(R)=$ $\sup \{\mathrm{C}-\operatorname{gldim}(T), \mathrm{C}-\operatorname{gldim}(D)\}$.

In other words, $R$ is $n$-perfect $(n \geq 0)$ if and only if $T$ and $D$ are $n$-perfect.

The proof of the theorem involves the following results, which are of independent interest.

Next lemma shows that the $n$-perfectness descends from a ring to any subring retract. Recall that, for a ring homomorphism $\psi: R \rightarrow S$, we say that $R$ is a subring retract of $S$, if there exists a ring homomorphism $\phi: S \longrightarrow R$ satisfying $\phi \psi=i d_{\mid R}$. In this case, $\psi$ is injective and the $R$-module $S$ contains $R$ as a direct summand [8, page 111].

Lemma 3.4 If $R$ is a subring retract of a ring $S$, then: $\mathrm{C}-\operatorname{gldim}(R) \leq \mathrm{C}-\operatorname{gldim}(S)$. 
Proof. we can assume that $\mathrm{C}-\operatorname{gldim}(S)=n$ for a positive integer $n$. Let $F$ be a flat $R$-module and let $N$ be an $R$-module. We have $\operatorname{Tor}_{p}^{R}(S, F)=0$ for all $p>0$. Then, from [4, Proposition 4.1.3],

$$
\operatorname{Ext}_{R}^{n+1}\left(F, N \otimes_{R} S\right) \cong \operatorname{Ext}_{S}^{n+1}\left(F \otimes_{R} S, N \otimes_{R} S\right)=0 .
$$

Since $R$ is a direct summand of the $R$-module $S, N$ is a direct summand of the $R$-module $N \otimes_{R} S$, and then $\operatorname{Ext}_{R}^{n+1}(F, N)$ is a direct summand of the $R$-module $\operatorname{Ext}_{R}^{n+1}\left(F, N \otimes_{R} S\right)$. Thus, $\operatorname{Ext}_{R}^{n+1}(F, N)=0$, as desired.

Lemma 3.5 Let $U$ be a multiplicative set of a ring $R$. For the ring of fractions $S=U^{-1} R$, we have: $\mathrm{C}-\operatorname{gldim}(S) \leq \mathrm{C}-\operatorname{gldim}(R)$.

Proof. We can assume that $\mathrm{C}-\operatorname{gldim}(R)=n$ for a positive integer $n$. Let $F$ be a flat $S$-module. Then, $F$ is a flat $R$-module, and so $\operatorname{pd}_{R}(F) \leq n$. Thus, there is an exact sequence of $R$-modules

$$
0 \rightarrow P_{n} \rightarrow \cdots \rightarrow P_{0} \rightarrow F \rightarrow 0
$$

where each $P_{i}$ is projective. Then, the sequence of $S$-modules

$$
0 \rightarrow P_{n} \otimes_{R} S \rightarrow \cdots \rightarrow P_{0} \otimes_{R} S \rightarrow F \otimes_{R} S=F \rightarrow 0
$$

is an exact sequence with each $P_{i} \otimes_{R} S$ is a projective $S$-module. This means that $S$ is $n$-perfect and therefore $\mathrm{C}-\operatorname{gldim}(S) \leq n$.

Proof of Theorem 3.3. First, note that $U^{-1} R=T$, where $U=D \backslash\{0\}$ (since $\mathrm{qf}(D)=K)$. Then, $\mathrm{C}-\operatorname{gldim}(T) \leq \mathrm{C}-\operatorname{gldim}(R)$ (by Lemma 3.5). And, from Lemma 3.4, $\mathrm{C}-\operatorname{gldim}(D) \leq \mathrm{C}-\operatorname{gldim}(R)$ (since $D$ is a subring retract of $R$ ).

It remains to prove the inequality $\mathrm{C}-\operatorname{gldim}(R) \leq \sup \{\mathrm{C}-\operatorname{gldim}(T), \mathrm{C}-\operatorname{gldim}(D)\}$. We can assume that $\mathrm{C}-\operatorname{gldim}(T) \leq n$ and $\mathrm{C}-\operatorname{gldim}(D) \leq n$ for a positive integer $n$. Let $F$ be a flat $R$-module. Consider the following exact sequence of $R$-modules $0 \rightarrow P \rightarrow P_{n-1} \rightarrow \cdots \rightarrow P_{0} \rightarrow F \rightarrow 0$, where each $P_{i}$ is projective. Decomposing this sequence into short exact sequences and applying successively [13, Exercise 8.2, page 223] (since $F$ is flat), we obtain the following exact sequences of $T$-modules and $R / M$-modules, respectively:

$$
\begin{gathered}
0 \rightarrow P \otimes_{R} T \rightarrow P_{n-1} \otimes_{R} T \rightarrow \cdots \rightarrow P_{0} \otimes_{R} T \rightarrow F \otimes_{R} T \rightarrow 0 \\
0 \rightarrow P / M P \rightarrow P_{n-1} / M P_{n-1} \rightarrow \cdots \rightarrow P_{0} / M P_{0} \rightarrow F / M F \rightarrow 0
\end{gathered}
$$

Since $\mathrm{C}-\operatorname{gldim}(T) \leq n$ and $\mathrm{C}-\operatorname{gldim}(D) \leq n, P \otimes_{R} S$ is a projective $S$-module and $P / M P$ is a projective $R / M$-module. Thus, $P$ is a projective $R$-module (by [8, Theorem 5.1.1(1)]), and then $\operatorname{pd}_{R}(F) \leq n$. This means that $R$ is $n$-perfect and therefore $\mathrm{C}-\operatorname{gldim}(R) \leq n$, which implies the desired inequality. 


\section{Applications and Examples}

Now, we are ready to give examples showing that each of the inequalities of Theorems 2.1 and 2.2 can be strict. We begin with two examples concerning Theorem 2.1. To this aim, we need the following result.

Proposition 4.1 ([6], Proposition 2.1) Let $V$ be a valuation domain of the form $K+M$, where $K$ is a field and $M(\neq 0)$ is a maximal ideal of $V$. Let $D$ be a proper subring of $K$ such that $\mathrm{qf}(D)=K$. For the pullback ring $R=D+M$, we have:

1. If $n=\operatorname{gldim}(V)$ and $m=\operatorname{gldim}(D)$, then:

$$
\operatorname{gldim}(R)= \begin{cases}n & \text { if } n>m ; \\ m & \text { if } m \geq n \text { and } \operatorname{pd}_{D}(K)<m ; \\ m+1 & \text { if } m \geq n \text { and } \operatorname{pd}_{D}(K)=m\end{cases}
$$

2. $\operatorname{wdim}(R)=\mathrm{wdim}(D)$.

The following example shows a ring $R$ of finite global dimension with the strict inequality $\mathrm{C}-\operatorname{gldim}(R)<\operatorname{gldim}(R)$ holds true.

Example 4.2 Let $\mathbb{Z}$ denote the ring of integers, and let $\mathbb{Q}$ denote the field of rational numbers. Consider the discrete valuation domain $V=\mathbb{Q}[X]_{(X)}=\mathbb{Q}+X V$ (the localization of the polynomial ring $\mathbb{Q}[X]$ at $(X))$. Then, for the pullback ring $R=$ $\mathbb{Z}+X V$, we have: $\operatorname{gldim}(R)=2, \operatorname{wdim}(R)=1$, and $\mathrm{C}-\operatorname{gldim}(R)=1$.

Proof. By Proposition 4.1, $\operatorname{wdim}(R)=\operatorname{wdim}(\mathbb{Z})=1$ and $\operatorname{gldim}(R)=2$ (since $\left.\operatorname{pd}_{\mathbb{Z}}(\mathbb{Q})=1\right)$.

From Theorem 3.3, $\mathrm{C}-\operatorname{gldim}(R)=\sup \{\mathrm{C}-\operatorname{gldim}(V), \mathrm{C}-\operatorname{gldim}(\mathbb{Z})\}=1$ (since $\operatorname{gldim}(V)=\operatorname{gldim}(\mathbb{Z})=1)$.

Note that, in Example 4.2 above, $\operatorname{gldim}(R)=\mathrm{C}-\operatorname{gldim}(R)+\operatorname{wdim}(R)$. This means that the upper bound on the global dimension given in Theorem 2.1 is the best possible upper bound.

In the next example we provide a ring $R$ of finite global dimension with the strict inequalities $\mathrm{C}-\operatorname{gldim}(R)<\operatorname{gldim}(R)<\mathrm{C}-\operatorname{gldim}(R)+\operatorname{wdim}(R)$ hold true.

Example 4.3 Let $\mathbb{C}$ denote the field of complex numbers and $\mathbb{C}(X, Y)$ denote the quotient field of the polynomial ring $\mathbb{C}[X, Y]$. Consider the discrete valuation domain $V=\mathbb{C}(X, Y)[Z]_{(Z)}=\mathbb{C}(X, Y)+Z V$ (the localization of the polynomial ring $\mathbb{C}(X, Y)[Z]$ at $(Z))$. Then, for the pullback ring $R=\mathbb{C}[X, Y]+Z V$, we have: $\operatorname{gldim}(R)=3$, $w \operatorname{dim}(R)=2$, and $\mathrm{C}-\operatorname{gldim}(R)=2$. 
Proof. By Proposition 4.1, $\operatorname{wdim}(R)=\operatorname{wdim}(\mathbb{C}[X, Y])=2$.

We have $2=\operatorname{gldim}(\mathbb{C}[X, Y])>\operatorname{gldim}(V)=1$ and $\operatorname{pd}_{\mathbb{C}[X, Y]}(\mathbb{C}(X, Y))=2$ ) (by [11, Theorem 2]). Then, from Proposition 4.1, $\operatorname{gldim}(R)=2+1=3$.

Since, $\mathrm{C}-\operatorname{gldim}(\mathbb{C}[X, Y])=2$ (by Theorem 3.1$)$, we obtain, from Theorem 3.3, that $\mathrm{C}-\operatorname{gldim}(R)=\sup \{\mathrm{C}-\operatorname{gldim}(\mathbb{C}[X, Y]), \mathrm{C}-\operatorname{gldim}(V)\}=2$, as desired.

Remark 4.4 Example 4.3 above answers the question evoked in Section 3, above Proposition 2.6.

Indeed, consider a valuation domain of the form $V=K+M$ and $R=D+M$. Let $Q$ denote the quotient field of $R$ and then of $V$. We have $\operatorname{pd}_{R}(Q)=\operatorname{pd}_{V}(Q)$ (this is obtained using the same argument as in the proof of the second part of Theorem 3.3 and since $Q \otimes_{R} V=Q$ and $Q \otimes_{R} R / M=0$ ).

Applying this fact to Example 4.3 above, we have $\operatorname{pd}_{R}(Q)=\operatorname{pd}_{V}(Q)=1$, and so $1=\operatorname{pd}_{R}(Q)<\mathrm{C}-\operatorname{gldim}(R)=2$.

Now we set examples concerning Theorem 2.2, To this aim, we use Theorem 3.2 and the fact that $\operatorname{gldim}(S \times R)=\sup \{\operatorname{gldim}(S), \operatorname{gldim}(R)\}$ for two $\operatorname{rings} R$ and $S$ [4. Chapitre VI, Exercise 8, page 123]. This result is a consequence of [12, Lemma $2.5(2)]$, which implies also that $\operatorname{FPD}(S \times R)=\sup \{\operatorname{FPD}(S), \operatorname{FPD}(R)\}$. Similarly, [2, Lemma 3.7] is used to prove that $\mathrm{wdim}(S \times R)=\sup \{\mathrm{wdim}(S), \mathrm{wdim}(R)\}$ and $\operatorname{FFD}(S \times R)=\sup \{\operatorname{FFD}(S), \operatorname{FFD}(R)\}$.

Next example shows a ring $R$ which has finite finitistic projective dimension, and infinite weak dimension and satisfies $\mathrm{C}-\operatorname{gldim}(R)<\operatorname{FPD}(R)=\mathrm{C}-\operatorname{gldim}(R)+$ $\operatorname{FFD}(R)$.

Example 4.5 Consider a perfect ring $S$ of infinite weak dimension. Then, for the ring $R$ of Example 4.2, we have: $\mathrm{C}-\operatorname{gldim}(S \times R)=1, \operatorname{FPD}(S \times R)=2$, $\operatorname{FFD}(S \times R)=1$, and $\mathrm{wdim}(S \times R)=\infty$.

Proof. Apply the facts mentioned above.

Using again the facts mentioned above, we get an example of a ring $R$ with the strict inequalities $\mathrm{C}-\operatorname{gldim}(R)<\operatorname{FPD}(R)<\mathrm{C}-\operatorname{gldim}(R)+\operatorname{FFD}(R)$ hold true.

Example 4.6 Consider a perfect ring $S$ of infinite weak dimension. Then, for the ring $R$ of Example 4.3, we have: $\mathrm{C}-\operatorname{gldim}(S \times R)=2, \operatorname{FPD}(S \times R)=3$, $\operatorname{FFD}(S \times R)=2$, and $\operatorname{wdim}(S \times R)=\infty$.

ACKNOWLEDGEMENTS. The authors would like to express their sincere thanks for the referee for his/her helpful suggestions. 
On $n$-Perfect Rings and Cotorsion Dimension

\section{References}

[1] H. Bass, Finitistic dimension and a homological generalization of semi-primary rings, Trans. Amer. Math. Soc. 95 (1960) 466-488.

[2] D. Bennis and N. Mahdou, Global Gorenstein dimensions of polynomial rings and of direct products of rings, Accepted for publication in Houston J. Math. Available from arXiv:0801.0483v1 (2008).

[3] N. Bourbaki, Algèbre Homologique, Chapitre 10 (Masson, Paris, 1980).

[4] H. Cartan and S. Eilenberg, Homological Algebra (Princeton University Press, 1956).

[5] N. Ding and L. Mao, The cotorsion dimension of modules and rings, Abelian groups, rings, modules, and homological algebra, Lect. Notes Pure Appl. Math., 249 (2005), 217-233.

[6] D. E. Dobbs, On the global dimensions of $D+M$, Canad. Math. Bull. 18 (1975) 657-660.

[7] E. E. Enochs, O. M. G. Jenda and J. A. López-Ramos, Dualizing modules and n-perfect rings, Proc. Edinb. Math. Soc. 48 (2005) 75-90.

[8] S. Glaz, Commutative Coherent Rings, Lect. Notes in Math. 1371 (SpringerVerlag, Berlin, Heidelberg, 1989).

[9] L. Gruson and M. Raynaud, Critères de platitude et de projectivité. Techniques de platification d'un module, Inv. Math. 13 (1971) 1-89.

[10] C. U. Jensen, On the vanishing of $\stackrel{(i)}{\longleftarrow}$, J.Algebra 15 (1970) 151-166.

[11] I. Kaplansky, The homological dimension of a quoient field, Nagoya Math. J. 27 (1966) 139-142.

[12] N. Mahdou, On Costa's conjecture, Comm. Algebra 29 (2001) 2775-2785.

[13] J. J. Rotman, An Introduction to Homological Algebra (Academic Press, London, New York, 1979).

[14] J. Xu, Flat Covers of Modules, Lect. Notes in Math. 1634 (Springer-Verlag, Berlin, Heidelberg, 1996). 